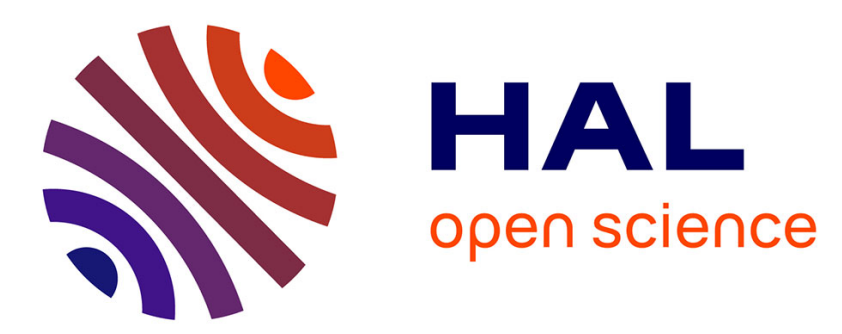

\title{
Peripubertal female athletes in high-impact sports show improved bone mass acquisition and bone geometry
} Laurent Maïmoun, Olivier Coste, Pascal Philibert, Karine Briot, Thibault

Mura, Florence Galtier, Denis Mariano-Goulart, Françoise Paris, Charles Sultan

\section{To cite this version:}

Laurent Maïmoun, Olivier Coste, Pascal Philibert, Karine Briot, Thibault Mura, et al.. Peripubertal female athletes in high-impact sports show improved bone mass acquisition and bone geometry. Metabolism, 2013, 10.1016/j.metabol.2012.11.010 . hal-02537284

\section{HAL Id: hal-02537284 \\ https://hal.umontpellier.fr/hal-02537284}

Submitted on 8 Apr 2020

HAL is a multi-disciplinary open access archive for the deposit and dissemination of scientific research documents, whether they are published or not. The documents may come from teaching and research institutions in France or abroad, or from public or private research centers.
L'archive ouverte pluridisciplinaire HAL, est destinée au dépôt et à la diffusion de documents scientifiques de niveau recherche, publiés ou non, émanant des établissements d'enseignement et de recherche français ou étrangers, des laboratoires publics ou privés. 


\title{
Peripubertal female athletes in high-impact sports show improved bone mass acquisition and bone geometry
}

\author{
Laurent Maïmoun ${ }^{a, b, *}$, Olivier Coste ${ }^{a, c}$, Pascal Philibert $^{a}$, Karine Briot $^{d}$, Thibault Mura $^{e, f}$, \\ Florence Galtier ${ }^{f, g}$, Denis Mariano-Goulart ${ }^{h}$, Françoise Paris ${ }^{a, i}$, Charles Sultan ${ }^{a, i}$ \\ a Département d'Hormonologie, Hôpital Lapeyronie, CHU Montpellier, 191 avenue Doyen Gaston Giraud, 34295 Montpellier, France \\ b Service de Médecine Nucléaire, Hôpital Lapeyronie, CHRU Montpellier, France and UMI and Physiologie \& médecine expérimentale du Cœur \\ et des Muscles - INSERM - U1046, Montpellier, France \\ ${ }^{c}$ Direction Régionale de la Jeunesse, des Sports et de la Cohésion Sociale, Montpellier, France \\ d Service de Rhumatologie, Hôpital Cochin, APHP Paris, France \\ e Centre d'Investigation Clinique et Département d'Information Médicale, CHRU Montpellier, Montpellier, France \\ ${ }^{\mathrm{f}}$ CIC 1001, INSERM, Montpellier, France \\ g Centre d'Investigation Clinique et Département des Maladies Endocriniennes, CHRU Montpellier, Montpellier, France \\ ${ }^{\mathrm{h}}$ Service de Médecine Nucléaire, Hôpital Lapeyronie, CHRU Montpellier, France \\ ${ }^{\text {i } U n i t e ́ ~ d ’ E n d o c r i n o l o g i e ~ P e ́ d i a t r i q u e, ~ H o ̂ p i t a l ~ A r n a u d ~ d e ~ V i l l e n e u v e, ~ C H R U ~ M o n t p e l l i e r ~ e t ~ U M I, ~ M o n t p e l l i e r, ~ F r a n c e ~}$
}

\section{A R T I C L E I N F O}

Keywords:

Bone mass acquisition

Intensive training

Growth period

Markers of bone turnover

OPG/RANKL system

\section{A B S T R A C T}

Objective. Intensive physical training may have a sport-dependent effect on bone mass acquisition. This cross-sectional study evaluated bone mass acquisition in girls practicing sports that put different mechanical loads on bone.

Materials/Methods. Eighty girls from 10.7 to 18.0 years old (mean 13.83 \pm 1.97 ) were recruited: 20 artistic gymnasts (AG; high-impact activity), 20 rhythmic gymnasts (RG; medium-impact activity), 20 swimmers (SW, no-impact activity), and 20 age-matched controls (CON; leisure physical activity $<3 \mathrm{~h} / \mathrm{wk}$ ). Areal bone mineral density (aBMD) was determined using DEXA. Hip structural analysis applied at the femur evaluated crosssectional area $\left(\mathrm{CSA}, \mathrm{cm}^{2}\right)$, section modulus $\left(\mathrm{Z}, \mathrm{cm}^{3}\right)$, and buckling ratio. Bone turnover markers and OPG/RANKL levels were analyzed.

Results. AG had higher aBMD than SW and CON at all bone sites and higher values than RG in the lumbar spine and radius. RG had higher aBMD than SW and CON only in the femoral region. CSA and mean cortical thickness were significantly higher and the buckling ratio was significantly lower in both gymnast groups compared with SW and CON. In RG only, endocortical diameter and width were reduced, while $Z$ was only increased in AG compared with SW and CON. Reduced bone remodeling was observed in RG compared with AG only when groups were subdivided according to menarcheal status. All groups showed similar OPG concentrations, while RANKL concentrations increased with age and were decreased in SW.

\footnotetext{
Abbreviations: aBMD, areal bone mineral density; AG, artistic gymnasts; BMI, body mass index; CON, controls; CSA, bone crosssectional area; CSMI, cross-sectional moment of inertia; CTX, type I-C telopeptide breakdown products; DEXA, dual-energy X-ray absorptiometry; FFST, fat-free soft tissue; FM, fat mass; HAS, hip structural analysis; IT, intertrochanteric; L1-L4, lumbar spine; NN, narrow-neck; OC, osteocalcin; OPG, osteoprotegerin; PINP, procollagen type I N-terminal propeptide; RANKL, rank-ligand; RG, rhythmic gymnasts; SW, swimmers; TPF, total proximal femur; WB, whole body; Z, section modulus.

* Corresponding author. Département d'Hormonologie, Hôpital Lapeyronie, CHU Montpellier, 191 avenue Doyen Gaston Giraud, 34295 Montpellier, France. Tel.: +33467 79 99; fax: +33 467338327.

E-mail addresses: 1-maimoun@chu-montpellier.fr, laurent.maimoun@laposte.net (L. Maïmoun).
} 
Conclusion. High-impact activity clearly had a favorable effect on aBMD and bone geometry during the growth period, although the bone health benefits seem to be more marked after menarche.

\section{Introduction}

Among the environmental factors that affect bone mass acquisition, physical activity plays a crucial role. Although its mode of action is not fully understood, it is generally assumed that mechanical loading is an important determinant of skeletal growth and modeling [1]. Weight-bearing activities are more beneficial for bone mass acquisition in childhood than no-impact and non-weight bearing activities $[2,3]$. These findings are mainly based on areal bone mineral density (aBMD) as evaluated by dual-energy x-ray absorptiometry (DEXA) scans, and less is known about the potential effects of exercise on bone microarchitecture and geometry, two parameters implicated in bone strength [4,5]. Studies using newer technologies, such as peripheral quantitative computed tomography (pQCT), which provides more detailed information about changes in cortical bone geometry and strength, are now available.

In adolescents, Sayers et al. [6] reported that regular physical activity like jogging was positively associated with cortical bone mass compared with less intense exercise like walking. The increase in cortical mass is due to increased periosteal and reduced endosteal circumferences, an adaptation that may improve mechanical bone strength [6]. Using an indirect method (hip structural analysis, HSA software) based on DXA analysis [7], we recently reported higher mean cortical thickness in the femur of young artistic gymnasts than in nonathlete subjects, and this bone adaptation was observed throughout the growth period [8]. However, artistic gymnastics generates high specific mechanical strains [9], and we do not currently know whether bone geometry gradually adapts to mechanical strain according to the type of sport, as previously reported for aBMD $[3,10,11]$. Moreover, the same aBMD may correspond to different bone geometry models. Ferry's study [12] provided some elucidation: they found a concomitant favorable effect on BMD and bone geometry in female adolescents playing soccer, a high-impact sport [11], as compared with swimming, a no-impact sport $[3,11]$.

These preliminary results on bone geometry should be confirmed in a range of sports generating different degrees of mechanical loading throughout the growth period, because age is a potential modulator of bone response [8,13]. A further consideration is that physical activity may also modulate bone remodeling $[8,14]$ and the osteoprotegerin (OPG)/rankligand (RANKL) system $[8,15,16]$, in addition to its effects on aBMD and bone geometry. These data are still controversial, however, and it remains to be demonstrated whether different types of sports generate specific profiles.

The aim of this cross-sectional study was to compare the bone mass acquisition in young girls performing sports that generate different mechanical loads. We included data from a population of artistic gymnasts [8], as this sport is a highimpact activity and the data helped us to determine the gradation in bone adaptation due to two other sports, rhythmic gymnastics (medium-impact) and swimming (lowimpact). The bone adaptation was concomitantly evaluated by bone mineral density, bone geometry at the femoral region, bone remodeling markers, and the OPG/RANKL system.

\section{Materials and methods}

\subsection{Subjects}

The study protocol was reviewed and approved by the Regional Research Ethics Committee (CPP Sud-Mediterranee IV, Montpellier, France), and each child and her parents gave written informed consent before entering the study. A total of 80 girls with ages ranging from 10.7 to 18.0 years (mean $13.83 \pm$ 1.97) were recruited for this study: 20 artistic gymnasts (AG), for whom part of the findings were previously reported [8], 20 rhythmic gymnasts (RG), 20 swimmers (SW), and 20 controls (CON). All athletes and controls were age-paired. The inclusion criteria were the following: The three sports groups were composed of girls training more than $8 \mathrm{~h}$ per week and who had been practicing their sport for more than 5 years. Each sport was chosen according to the mechanical loading that it generates (high for AG, medium for RG, and low for SW). Moreover, although AG and RG seem to be very similar sports (high-volume training, high prevalence of menstrual disorders), they differ in terms of the intensity and sites of the mechanical loading, as well as the individual growth potential $[17,18]$. The control group consisted of subjects who performed only leisure physical activities for fewer than $3 \mathrm{~h}$ per week, and none of these subjects had a history of participation in intense sports training.

The exclusion criteria were as follows: obvious signs of acute or chronic illness known to affect bone health and long periods of immobilization or fractures within the previous 12 months.

\subsection{Methods}

The design of this cross-sectional and case-control study has been described in detail [8]. Standing height was measured with a stadiometer to the nearest $0.1 \mathrm{~cm}$. Weight was determined using a weight scale with a precision of $0.1 \mathrm{~kg}$. Body mass index (BMI) was calculated as weight (kg) divided by the square of height $(\mathrm{m})$. Pubertal development was assessed by breast stage (I to V) according to the Tanner classification [19] by an experienced pediatric endocrinologist. Skeletal age was determined using the Greulich and Pyle method [20].

Information regarding pubertal onset in family members was obtained from a standardized questionnaire (menarche of mothers). Height standard deviation score (height SDS) and 
weight standard deviation score (weight SDS) were calculated according to the French standard curves. Moreover, target height, which is the expected final height of a child given the adult heights of both parents, was calculated with the following equation:

Target height $=([$ father's height + mother's height $] / 2)-6.5$.

[19].

\subsubsection{Medical and menstrual histories}

[19] Each subject or her parents responded to a medical questionnaire designed to assess the general medical and menstrual history, with questions on the age of menarche and the presence of menstrual disorders.

\subsubsection{Physical activity determination}

Detailed information about training history was collected, including data on starting age of intensive training, years of active sport-specific training, and number of training sessions per week, training hours per week, and training months per year. Other physical activities were documented with a training recall diary covering the previous 3 years.

\subsubsection{Assays}

Blood samples $(25 \mathrm{ml})$ were collected in the morning (090011 h00) in sterile chilled tubes by standard venipuncture technique. The samples were allowed to clot at room temperature and were then centrifuged at $2500 \mathrm{rpm}$ for $10 \mathrm{~min}$ at $4{ }^{\circ} \mathrm{C}$. Plasma samples were stored at $-80^{\circ} \mathrm{C}$ until analysis. All samples were run in duplicate and, to reduce inter-assay variation, all the plasma samples were analyzed in a single session. In the pubertal girls, the date of the last menses was not recorded and hormonal values were thus obtained at an unsynchronized menstrual stage.

Concerning bone metabolism, plasma samples were assayed by Cobas 6000 (Roche Diagnostic, Mannheim, Germany) for osteocalcin (OC), procollagen type I N-terminal propeptide (PINP), and type I-C telopeptide breakdown products (CTX). The inter- and intra-assay CVs for the three parameters were lower than $7 \%$.

The intra- and inter-assay CVs for OPG were, respectively, 10\% and 7\% (OPG ELISA, Immunodiagnostic Systems, Boldon, UK); for RANKL (ampli sRANKL human ELISA, Immunodiagnostic Systems, Boldon, UK) they were, respectively, $8 \%$ and $6 \%$.

2.2.4. Bone mineral density, body fat and fat-free soft tissues DXA (Hologic QDR-4500A, Hologic, Waltham, MA) measured the areal bone mineral density $\left(\mathrm{BMD} ; \mathrm{g} / \mathrm{cm}^{2}\right)$ of the whole body (WB) and at specific bone sites: the antero-posterior lumbar spine (L1-L4), the dominant arm radius, the proximal part of the left femur (TPF), and specific femoral sites: the neck and the trochanteric and intertrochanteric areas. The soft tissue body composition (fat mass [FM, kg], percentage of body fat mass [\% FM] and fat-free soft tissue [FFST, kg]) was derived from the whole body scan. All scanning and analyses were performed by the same operator to ensure consistency, after following standard quality control procedures. Quality control for DXA was checked daily by scanning a lumbar spine phantom consisting of calcium hydroxyapatite embedded in a cube of thermoplastic resin (DPA/QDR-1; Hologic x-caliber anthropometric spine phantom). For BMD, the laboratory precision error was defined by the CV of repeated measurements; this was found to be $1 \%$ at the lumbar spine and $<1 \%$ at the femoral neck, $<1 \%$ at the forearm, $<0.5 \%$ for the whole body, and $<1 \%$ for FFST and FM.

\subsubsection{Bone geometry of the proximal femur}

Using DXA data, we analyzed hip geometry parameters with HSA software [7]. The HSA program uses mineral mass and dimensional data from conventional DXA images of the hip to measure the structural dimensions of bone cross-sections corresponding to three thin regions traversing the proximal femur: the narrow-neck (NN) region across the narrowest point of the femoral neck, the intertrochanteric (IT) region across the bisector of the neck and shaft axes, and the shaft region located at a distance 1.5 times the width of the femoral neck distal to the intersection of the neck and shaft axes. For each region, the distribution of the bone mass across the bone was extracted and the following measurements were made directly from the bone mass profile: (1) the subperiosteal width (width, cm); (2) the bone cross-sectional area exclusive of soft-tissue spaces (CSA; in $\mathrm{cm}^{2}$ ), an index of resistance to axial forces; and (3) the cross-sectional moment of inertia (CSMI, $\mathrm{cm}^{4}$ ). In addition, (4) the endosteal diameter was estimated using the algorithm described by Beck et al. [7], (5) the mean cortical thickness was calculated as the difference between the periosteal and endosteal diameters divided by two, and (6) the section modulus $(\mathrm{Z})\left(\mathrm{cm}^{3}\right)$, an index of bone binding strength, was calculated $(Z=C S M I / y)$, where $y=1 / 2$ subperiosteal width for the neck and shaft regions and the distance from the centroid to the lateral cortical margin for the intertrochanteric region. Last, (7) the buckling ratio, an index of susceptibility to local cortical buckling under compressive loads, was calculated as the distance from the center of mass to the medial or lateral cortex divided by the estimated average cortical thickness. HSA precision has not yet been assessed in children and adolescents; however, experience in mainly elderly populations has shown HSA precision on the order of $1 \%-5 \%$ for the HSA parameters at the three HSA regions [21]. All the values were also adjusted to body weight and body height, as recommended by Beck et al. [7]. A single physician conducted all software analyses.

\section{Statistical analysis}

The characteristics of the young athletes and controls are described with proportions for categorical variables and with means and standard deviation values for continuous variables (age, weight, aBMD, etc.). The distributions were tested with the Shapiro-Wilk statistic. The comparisons of means between the gymnasts, swimmers and controls (and between subgroups according to the menarcheal status in order to explore the interaction between menarcheal status and sport) were performed using ANOVA when data distribution was normal and the Kruskall-Wallis test if continuous variables were skewed. For each BMD site, adjusted means for age, FM and FFST were computed and compared between groups using multivariate linear regression analysis. Family-wise 
error rates were controlled using the Ryan (REGWQ for crude analysis) and Tukey (for adjusted analysis) multiple comparison procedures.

Statistical analyses were performed at the conventional two-tailed $\alpha$ level of 0.05 using SAS version 9.1 (SAS Institute, Cary, NC).

\section{Results}

The anthropometric characteristics and training status of the athletes and controls are summarized in Table 1. The age distribution ranged from 10.7 to 18.0 years, with a mean age of $13.8 \pm 2.0$ years, and all athletes and controls were age-paired. There were no significant differences between groups with regard to weight, BMI or body fat-free soft tissue, while body fat mass (kg) was significantly lower in both groups of gymnasts compared with SW and CON. AG and RG were shorter than SW. When height SDS and weight SDS were calculated according to the French standard curves, AG presented lower values than CON, and AG and RG had lower values than SW. No difference was observed for bone age between the four groups; however, when the difference between chronological age and bone age ( $\Delta$ age - bone age) was calculated, AG and RG presented significantly higher values than SW and CON. The age of menarche was significantly delayed in AG $(13.7 \pm 1.2 \mathrm{yr})$ and RG $(14.2 \pm 1.5 \mathrm{yr})$ compared with CON $(12.1 \pm 0.9 \mathrm{yr})$ but did not differ from that in SW $(13.2 \pm 1.3 \mathrm{yr})$. Moreover, in RG, two adolescents presented no menarche at 14.1 years, suggesting that the final mean age of menarche for this group was greater than 14.2 years. The athletes presented a high frequency of menstrual disorders (50\%-63.3\%) compared with the controls.
Target height was lower in AG $(p=0.002)$ compared with the other three groups. No significant differences in mean Tanner stages of breast development were noted between groups.

On average, the total number of training hours per week was higher in both gymnast groups compared with SW, but no difference in the age at the start of training was observed between trained groups. CON were normally active and participated only in leisure physical activities for fewer than $3 \mathrm{~h}$ per week. The CON activities included rhythmic and artistic gymnastics, tennis, judo, volleyball, basketball, handball, judo and karate.

\subsection{Bone characteristics}

\subsubsection{Areal bone mineral density}

Table 2 presents the unadjusted aBMD for the four groups at various bone sites. Compared with SW and CON, AG presented a noticeably greater aBMD at all bone sites (TPF, L2-L4 and radius), skull and WB excepted. The differences between CON and AG ranged between $8.9 \%$ for WB and 19.1\% for the femoral neck. The two gymnastics groups differed only in the radius and lumbar spine aBMD, with AG presenting higher values than RG. For RG, BMD was higher than for SW and CON only in the femoral region. No difference was observed between SW and CON for any bone site.

In parallel with the results for the unadjusted BMDs, the adjusted BMDs for age, and the FFST and FM values measured at the femoral region, L2-L4 and the radius were higher in the AG group, skull excepted, compared with the SW and CON groups (Table 3). The difference between AG and RG persisted at the radius and lumbar spine. Minor differences compared with unadjusted BMD values were observed, including higher WB values in both groups of gymnasts compared with SW and CON, higher values at the pelvis in AG compared with SW and

\begin{tabular}{|c|c|c|c|c|c|}
\hline Parameters & Artistic Gymnasts & Rhythmic Gymnasts & Swimmers & Controls & p-value \\
\hline Number of subjects & 20 & 20 & 20 & 20 & \\
\hline Age (yr) & $13.8 \pm 2.0$ & $13.8 \pm 2.2$ & $14.1 \pm 1.8$ & $13.7 \pm 2.0$ & 0.854 \\
\hline Bone age (yr) & $13.1 \pm 2.3$ & $12.4 \pm 2.5$ & $13.9 \pm 2.0$ & $13.7 \pm 2.4$ & 0.136 \\
\hline$\Delta$ Age - bone age (yr) & $0.7 \pm 1.0^{*}$ & $1.4 \pm 1.1^{* *}, \dagger$ & $0.1 \pm 1.0$ & $0.1 \pm 1$ & $<0.001$ \\
\hline \multicolumn{6}{|l|}{ Tanner stages } \\
\hline (number per stage I, II, III, IV, V) & $4 ; 1 ; 2 ; 1 ; 12$ & $3 ; 7 ; 3 ; 0 ; 7$ & $2 ; 2 ; 1 ; 5 ; 10$ & $2 ; 3 ; 3 ; 1 ; 11$ & 0.103 \\
\hline Age of menarche (yr) & $13.7 \pm 1.2^{* *}$ & $14.2 \pm 1.5^{* *}$ & $13.2 \pm 1.3$ & $12.1 \pm 0.9$ & 0.004 \\
\hline Number of subjects with menarche & $11(55 \%)$ & $6(30 \%)$ & $11(55 \%)$ & $12(60 \%)$ & 0.221 \\
\hline Number of subjects with menstrual disorders & $7(63.6 \%)$ & $3(50 \%)$ & $6(54.5 \%)$ & $0(100 \%)$ & \\
\hline \multicolumn{6}{|l|}{ Anthropometric data } \\
\hline Weight $(\mathrm{kg})$ & $45.4 \pm 8.6$ & $43.6 \pm 9.0$ & $50.1 \pm 6.1$ & $47.0 \pm 10.8$ & 0.118 \\
\hline Weight SDS & $-0.02 \pm 0.7$ & $-0.06 \pm 0.6$ & $0.6 \pm 0.9$ & $0.4 \pm 1.3$ & 0.044 \\
\hline Height $(\mathrm{cm})$ & $151.4 \pm 8.0^{\dagger+\dagger}$ & $153.5 \pm 9.5^{\dagger+}$ & $162.2 \pm 4.7$ & $156.5 \pm 8.7$ & $<0.001$ \\
\hline Height SDS & $-0.6 \pm 0.7^{* *},+\dagger$ & $-0.1 \pm 0.9^{\dagger \dagger}$ & $1.0 \pm 0.8$ & $0.45 \pm 0.9$ & $<0.001$ \\
\hline BMI $\left(\mathrm{kg} \mathrm{m}^{-2}\right)$ & $19.6 \pm 2.0$ & $18.3 \pm 2.0$ & $19.0 \pm 1.9$ & $19.0 \pm 3.0$ & 0.252 \\
\hline Target height $(\mathrm{cm})$ & $160.4 \pm 4.2^{*}, t+t, \neq$ & $165.8 \pm 4.2$ & $165.3 \pm 3.9$ & $163.9 \pm 4.5$ & $<0.001$ \\
\hline Body fat mass (kg) & $7.7 \pm 2.1^{*},+$ & $8.6 \pm 2.9^{*}$, & $10.3 \pm 3.0$ & $11.2 \pm 4.8$ & 0.011 \\
\hline Body fat mass (\%) & $16.9 \pm 2.3^{*},+t+$ & $19.4 \pm 3.7^{*}$ & $20.4 \pm 4.6$ & $22.8 \pm 5.1$ & $<0.001$ \\
\hline Body fat-free soft tissue (kg) & $35.8 \pm 6.4$ & $33.3 \pm 6.2$ & $38.1 \pm 4.3$ & $34.3 \pm 6.2$ & 0.064 \\
\hline \multicolumn{6}{|l|}{ Training status } \\
\hline Hours/weeks & $20.3 \pm 4.2^{* * *}$, & $21.1 \pm 4.4^{* * *}, \dagger$ & $14.5 \pm 5.9^{* * *}$ & $2.5 \pm 0.5^{c}$ & $<0.001$ \\
\hline Age at start of training (yr) & $5.6 \pm 1.7$ & $6.6 \pm 1.2$ & $6.5 \pm 1.8$ & - & - \\
\hline
\end{tabular}

Values are presented as mean \pm SD. BMI: body mass index. ${ }^{*} p<0.05,{ }^{* *} p<0.01$, and ${ }^{* * *} p<0.001$ denote a significant difference with controls; $\dagger p<0.05$, $\dagger+p<0.01$, and $+\dagger+p<0.001$ denote a significant difference with swimmers; $\neq p<0.001$ denotes a significant difference with rhythmic gymnasts. 
Table 2 - Areal bone mineral density at various bone sites in athletes and controls.

\begin{tabular}{|c|c|c|c|c|c|}
\hline Bone mineral density $\left(\mathrm{g} \mathrm{cm}^{-2}\right)$ & Artistic Gymnasts & Rhythmic Gymnasts & Swimmers & Controls & $\mathrm{p}$-value \\
\hline Whole body & $1.035 \pm 0.113$ & $0.992 \pm 0.132$ & $0.958 \pm 0.079$ & $0.950 \pm 0.091$ & 0.073 \\
\hline Arm BMD & $0.751 \pm 0.088^{* * *, t \mathrm{t}, \neq \neq}$ & $0.651 \pm 0.072$ & $0.695 \pm 0.016$ & $0.653 \pm 0.060$ & $<0.001$ \\
\hline Leg BMD & $1.094 \pm 0.148^{*},+$ & $1.028 \pm 0.153$ & $0.992 \pm 0.074$ & $0.979 \pm 0.094$ & 0.019 \\
\hline Pelvis BMD & $1.167 \pm 0.183$ & $1.085 \pm 0.211$ & $1.048 \pm 0.123$ & $1.038 \pm 0.131$ & 0.091 \\
\hline Skull BMD & $1.685 \pm 0.197$ & $1.768 \pm 0.332$ & $1.676 \pm 0.206$ & $1.698 \pm 0.322$ & 0.919 \\
\hline \multicolumn{6}{|l|}{ Femoral region } \\
\hline Femoral neck & $0.908 \pm 0.142^{* * *,+\dagger}$ & $0.930 \pm 0.192^{* *},+\dagger$ & $0.764 \pm 0.077$ & $0.762 \pm 0.100$ & $<0.001$ \\
\hline Trochanter & $0.775 \pm 0.140^{*}$ & $0.774 \pm 0.155^{*}$ & $0.688 \pm 0.086$ & $0.671 \pm 0.095$ & 0.010 \\
\hline Intertrochanteric region & $1.104 \pm 0.190^{*}$, & $1.070 \pm 0.209$ & $0.975 \pm 0.141$ & $0.961 \pm 0.117$ & 0.019 \\
\hline Total proximal femur & $0.974 \pm 0.159^{*}, \dagger$ & $0.969 \pm 0.183^{*}, \dagger$ & $0.855 \pm 0.105$ & $0.857 \pm 0.105$ & 0.007 \\
\hline Lumbar spine (L1-L4) & $0.946 \pm 0.158^{*},+, \neq$ & $0.831 \pm 0.179$ & $0.822 \pm 0.100$ & $0.835 \pm 0.130$ & 0.026 \\
\hline Radius & $0.548 \pm 0.077^{*},+, \neq \neq$ & $0.482 \pm 0.060$ & $0.474 \pm 0.048$ & $0.484 \pm 0.051$ & 0.004 \\
\hline
\end{tabular}

Values are presented as mean $\pm S D .{ }^{*} p<0.05,{ }^{* *} p<0.01$, and ${ }^{* * *} p<0.001$ denote a significant difference with controls; $\uparrow p<0.05, \dagger+p<0.01$, and $\dagger+\uparrow \mathrm{p}<0.001$ denote a significant difference with swimmers; $\neq \mathrm{p}<0.05$ and $\neq \neq \mathrm{p}<0.001$ denote a significant difference with rhythmic gymnasts.

CON, lower values at L1-L4 in SW compared with RG, and lower values at the radius in SW compared with CON.

To determine the effect of menarche on aBMD, the four groups were subdivided according to menarcheal status. For all bone sites and for each group, unadjusted aBMD was significantly higher during the postmenarcheal period compared with the premenarcheal period (data not shown). Nevertheless, as menarcheal age differed between groups, aBMD was adjusted by age. AG and RG presented higher aBMD than SW and CON only during the postmenarcheal period. A significant interaction between menarcheal status and sport was found at the total proximal femur and lumbar spine for RG and at the radius for AG (Fig. 1).

\subsubsection{Bone geometry}

The between-group comparisons of bone geometry at the proximal femur are presented in Table 4. After adjustment for height and weight, the calculated values in the three femoral regions of neck (FN), intertrochanteric region (IR) and shaft (S) were significantly higher for CSA (except at S for RG) and mean cortical thickness, and significantly lower for buckling ratio (except at FN), in both groups of gymnasts compared with SW and CON. Endocortical diameter and width (to a lesser extent) were reduced in RG, but the statistical difference with the other three groups varied according to the femoral region. $\mathrm{Z}$ was only increased in AG compared with SW and CON, at the three regions. No difference was observed between groups for CSMI. No difference between SW and CON was observed whatever the parameter calculated or the femoral region.

\subsubsection{Bone biochemical markers and the OPG/RANKL system} Concerning the biochemical markers of bone turnover (Table 5), no significant difference for markers of bone formation (OC, P1NP) was observed between groups, while a marker of bone resorption (CTx) tended to be reduced $(p=0.06)$ in RG compared with AG. When parameters adjusted to age were analyzed in subgroups according to menarcheal status, RG presented lower values $(p<0.0014)$ than AG for OC in the premenarcheal period. No significant difference was observed for PINP and sCTX between the four groups (Fig. 2A,C). All the bone biochemical markers: OC, PINP, and CTX, decreased significantly with age and values in the premenarcheal period were found to be significantly higher than in the postmenarcheal period (data not shown). Moreover, the lack of

Table 3 - Adjusted areal bone mineral density by age, body fat mass and body fat-free soft tissue at various bone sites in athletes and controls.

\begin{tabular}{|c|c|c|c|c|c|}
\hline Bone mineral density $\left(\mathrm{g} \mathrm{cm}^{-2}\right)$ & Artistic Gymnasts & Rhythmic Gymnasts & Swimmers & Controls & $\mathrm{p}$-value \\
\hline Whole body & $1.031 \pm 0.013^{* *},+\dagger$ & $1.012 \pm 0.013^{* *},+\dagger$ & $0.929 \pm 0.013$ & $0.923 \pm 0.013$ & $<0.0001$ \\
\hline Arm BMD & $0.748 \pm 0.010^{* * *}, t+\mathrm{t}, \neq \neq$ & $0.666 \pm 0.010$ & $0.673 \pm 0.010$ & $0.661 \pm 0.010$ & $<0.0001$ \\
\hline Leg BMD & $1.090 \pm 0.014^{* * *},+十$ & $1.059 \pm 0.013^{* *}$ & $0.951 \pm 0.014$ & $0.993 \pm 0.014$ & $<0.0001$ \\
\hline Pelvis BMD & $1.165 \pm 0.023^{* *}$ & $1.124 \pm 0.022^{\dagger+}$ & $0.995 \pm 0.022$ & $1.053 \pm 0.024$ & $<0.0001$ \\
\hline Skull BMD & $1.676 \pm 0.045$ & $1.787 \pm 0.043$ & $1.639 \pm 0.044$ & $1.725 \pm 0.045$ & 0.123 \\
\hline \multicolumn{6}{|l|}{ Femoral region } \\
\hline Femoral neck & $0.909 \pm 0.022^{* * *}, \mathrm{t \dagger}$ & $0.957 \pm 0.021^{* * *},+\dagger+$ & $0.728 \pm 0.02$ & $0.769 \pm 0.022$ & $<0.0001$ \\
\hline Trochanter & $0.771 \pm 0.020^{*},+\dagger$ & $0.801 \pm 0.019^{* *},+t+$ & $0.652 \pm 0.020$ & $0.684 \pm 0.021$ & $<0.0001$ \\
\hline Intertrochanteric region & $1.106 \pm 0.026^{* *}, \mathrm{tt}$ & $1.102 \pm 0.024^{* *, t \dagger}$ & $0.930 \pm 0.025$ & $0.972 \pm 0.026$ & $<0.0001$ \\
\hline Total proximal femur & $0.972 \pm 0.020^{* *}$ & $0.998 \pm 0.020^{* * *},+\dagger+$ & $0.814 \pm 0.020$ & $0.870 \pm 0.021$ & $<0.0001$ \\
\hline Lumbar spine (L1-L4) & $0.943 \pm 0.018^{* *}, \mathrm{tt+}, \neq$ & $0.858 \pm 0.018^{\dagger}$ & $0.782 \pm 0.018$ & $0.850 \pm 0.019$ & $<0.0001$ \\
\hline Radius & $0.552 \pm 0.008^{* * *}, t+t, \neq \neq$ & $0.491 \pm 0.008$ & $0.461 \pm 0.008^{*}$ & $0.495 \pm 0.009$ & $<0.0001$ \\
\hline
\end{tabular}

Values are presented as mean $\pm S D .{ }^{*} p<0.05,{ }^{* *} p<0.01$, and ${ }^{* * *} p<0.001$ denote a significant difference with controls; $\uparrow p<0.05, \uparrow+p<0.01$, and $+\uparrow+p<0.001$ denote a significant difference with swimmers; $\neq \mathrm{p}<0.01$ and $\neq \neq \mathrm{p}<0.001$ denote a significant difference with rhythmic gymnasts. 

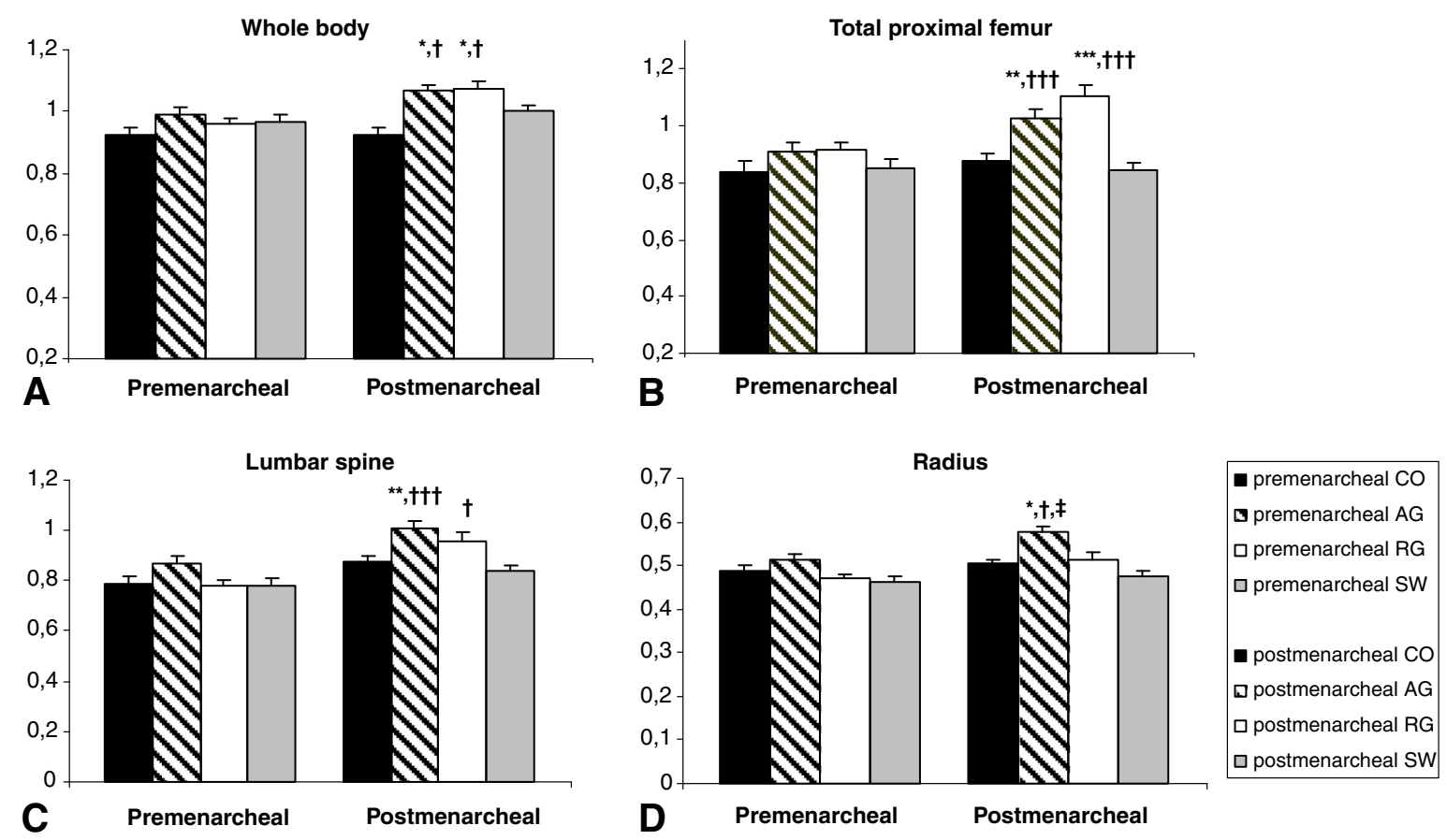

Fig. 1 - Adjusted aBMD by age in athletes and controls according to menarcheal status. Values are presented as means \pm SEM. aBMD at whole body (A), total proximal femur (B), lumbar spine (C) and radius (D). ${ }^{*} \mathrm{p}<0.05$ denotes a significant difference with controls; $\uparrow p<0.05, \dagger+p<0.01$ denotes a significant difference with swimmers; $\neq p<0.05$ denotes a significant difference with rhythmic gymnasts.

Table 4 - Hip structural analysis (HSA) parameters adjusted for body weight and body height.

\begin{tabular}{|c|c|c|c|c|}
\hline Parameters & Artistic Gymnasts & Rhythmic Gymnasts & Swimmers & Controls \\
\hline HAL (mm) & $107.7 \pm 1.2$ & $105.9 \pm 1.1$ & $108.2 \pm 1.2$ & $104.7 \pm 1.1$ \\
\hline Shaft neck angle $\left(^{\circ}\right)$ & $131.2 \pm 1.2$ & $131.1 \pm 1.2$ & $131.2 \pm 1.2$ & $130.1 \pm 1.1$ \\
\hline \multicolumn{5}{|l|}{ Femoral neck } \\
\hline Cross-sectional area $\left(\mathrm{CSA} ; \mathrm{cm}^{2}\right)$ & $3.085 \pm 0.083^{* * *},+\dagger$ & $2.936 \pm 0.080^{* * *},+$ & $2.460 \pm 0.082$ & $2.610 \pm 0.073$ \\
\hline Cross-sectional moment of inertia (CSMI; $\left.\mathrm{cm}^{4}\right)$ & $2.083 \pm 0.091$ & $1.808 \pm 0.088$ & $1.745 \pm 0.091$ & $1.875 \pm 0.084$ \\
\hline Section modulus $\left(Z ; \mathrm{cm}^{3}\right)$ & $1.424 \pm 0.046^{* *}, \mathrm{t \dagger}$ & $1.286 \pm 0.044^{\dagger}$ & $1.114 \pm 0.046$ & $1.220 \pm 0.042$ \\
\hline Endocortical diameter $(\mathrm{cm})$ & $2.359 \pm 0.057^{\dagger}$ & $2.227 \pm 0.055^{\dagger \dagger}$ & $2.584 \pm 0.057$ & $2.518 \pm 0.052$ \\
\hline Buckling ratio & $6.574 \pm 0.362^{* *, t \dagger}$ & $6.338 \pm 0.349^{* * *}$, & $9.030 \pm 0.362$ & $8.279 \pm 0.333$ \\
\hline Mean cortical thickness $(\mathrm{cm})$ & $0.229 \pm 0.007^{* * *, t \dagger}$ & $0.230 \pm 0.007^{* * *,+\dagger}$ & $0.170 \pm 0.007$ & $0.184 \pm 0.007$ \\
\hline Width $(\mathrm{cm})$ & $2.816 \pm 0.050$ & $2.686 \pm 0.049^{*},+\dagger$ & $2.924 \pm 0.050$ & $2.889 \pm 0.046$ \\
\hline \multicolumn{5}{|l|}{ Intertrochanteric region } \\
\hline Cross-sectional area $\left(\mathrm{CSA} ; \mathrm{cm}^{2}\right)$ & $4.950 \pm 0.138^{* * *,+\dagger \dagger}$ & $4.706 \pm 0.133^{* *},+\dagger+$ & $3.908 \pm 0.138$ & $4.148 \pm 0.127$ \\
\hline Cross-sectional moment of inertia (CSMI; $\left.\mathrm{cm}^{4}\right)$ & $9.319 \pm 0.425^{\dagger, \ddagger}$ & $7.795 \pm 0.408$ & $7.339 \pm 0.424$ & $8.146 \pm 0.90$ \\
\hline Section modulus $\left(Z ; \mathrm{cm}^{3}\right)$ & $3.556 \pm 0.130^{* *}, t+$ & $3.148 \pm 0.126$ & $2.834 \pm 0.130$ & $2.942 \pm 0.120$ \\
\hline Endocortical diameter $(\mathrm{cm})$ & $3.810 \pm 0.088^{\ddagger}$ & $3.473 \pm 0.084^{* *}, \dagger$ & $3.910 \pm 0.087$ & $4.027 \pm 0.080$ \\
\hline Buckling ratio & $5.732 \pm 0.259^{* *}$ & $5.289 \pm 0.249^{* * *},+\dagger$ & $6.893 \pm 0.258^{b}$ & $6.884 \pm 0.238^{b}$ \\
\hline Mean cortical thickness (cm) & $0.472 \pm 0.015^{* *}$ & $0.472 \pm 0.014^{* *},+\dagger \dagger$ & $0.371 \pm 0.015$ & $0.394 \pm 0.013$ \\
\hline Width $(\mathrm{cm})$ & $4.753 \pm 0.081^{\ddagger}$ & $4.418 \pm 0.080^{* *}$ & $4.653 \pm 0.081$ & $4.816 \pm 0.075$ \\
\hline \multicolumn{5}{|l|}{ Femoral shaft } \\
\hline Cross-sectional area $\left(\mathrm{CSA} ; \mathrm{cm}^{2}\right)$ & $3.714 \pm 0.095^{* *},+\dagger$ & $3.530 \pm 0.091^{\dagger \dagger}$ & $3.109 \pm 0.095$ & $3.266 \pm 0.088$ \\
\hline Cross-sectional moment of inertia (CSMI; $\mathrm{cm}^{4}$ ) & $2.406 \pm 0.118^{\dagger}$ & $2.062 \pm 0.114$ & $1.933 \pm 0.118$ & $2.054 \pm 0.109$ \\
\hline Section modulus $\left(Z ; \mathrm{cm}^{3}\right)$ & $1.731 \pm 0.060^{*},+\dagger$ & $1.569 \pm 0.058$ & $1.422 \pm 0.060$ & $1.499 \pm 0.055$ \\
\hline Endocortical diameter $(\mathrm{cm})$ & $1.414 \pm 0.085$ & $1.265 \pm 0.081^{\dagger}$ & $1.602 \pm 0.085$ & $1.522 \pm 0.078$ \\
\hline Buckling ratio & $2.437 \pm 0.143$ & $2.244 \pm 0.137^{\dagger}$ & $2.832 \pm 0.143$ & $2.661 \pm 0.132$ \\
\hline Mean cortical thickness $(\mathrm{cm})$ & $0.601 \pm 0.026^{*}$, & $0.608 \pm 0.025^{*}$, & $0.478 \pm 0.026$ & $0.513 \pm 0.024$ \\
\hline Width $(\mathrm{cm})$ & $2.616 \pm 0.047$ & $2.481 \pm 0.046$ & $2.557 \pm 0.047$ & $2.547 \pm 0.043$ \\
\hline
\end{tabular}

Values are presented as mean \pm SEM. ${ }^{*} p<0.05,{ }^{* *} p<0.01$, and ${ }^{* * *} p<0.001$ denote a significant difference with controls; $\dagger p<0.05,+\dagger p<0.01$, and $\dagger+\mathrm{t}<0.001$ denote a significant difference with swimmers; $\neq \mathrm{p}<0.05$ denotes a significant difference with rhythmic gymnasts. 
Table 5 - Bone biochemical markers and OPG/RANKL system in gymnasts and controls.

\begin{tabular}{lcccc} 
Parameters & Artistic Gymnasts & Rhythmic Gymnasts & Swimmers & Controls \\
\hline Bone biochemical markers & & & & \\
PINP $\left(\mathrm{ng} \mathrm{ml}^{-1}\right.$ ) & $581.5 \pm 349.2$ & $443.2 \pm 307.1$ & $463.1 \pm 327.0$ & $439.2 \pm 322.5$ \\
OC $\left(\mathrm{ng} \mathrm{ml}^{-1}\right.$ ) & $133.2 \pm 78.9$ & $77.6 \pm 39.0$ & $94.8 \pm 46.6$ & $94.1 \pm 55.6$ \\
CTx $\left(\mathrm{ng} \mathrm{ml}^{-1}\right.$ ) & $1.413 \pm 0.663$ & $0.930 \pm 0.470$ & $1.216 \pm 0.641$ & $1.053 \pm 0.513$ \\
OPG $\left(\mathrm{pmol} \mathrm{l}^{-1}\right.$ ) & $3.5 \pm 0.7$ & $3.1 \pm 0.8$ & $3.0 \pm 0.7$ & 0.470 \\
RANKL $\left(\mathrm{pmol} \mathrm{l}^{-1}\right.$ ) & $0.51 \pm 0.25^{\dagger}$ & $0.56 \pm 0.33^{\dagger}$ & $0.32 \pm 0.32$ & 0.057 \\
\hline
\end{tabular}

Values are presented as means \pm SD; PINP: procollagen type I N-terminal propeptide; OC: osteocalcin; CTx: type I-C telopeptide breakdown products, OPG: osteoprotegerin; RANKL: rank-ligand. $\uparrow \mathrm{p}<0.05$ denotes a significant difference with swimmers.

interaction between menarcheal status and sport indicated a similar profile for bone remodeling between the groups.

No between-group difference for OPG concentrations was observed (Table 4), whatever the menarcheal status (Fig. 2D). RANKL concentrations were significantly decreased in SW than in the three other groups and this was mainly due to a lower value in the postmenarcheal period (Fig. 2E). An interaction between menarcheal status and sport was observed only in RG and SW. RANKL concentrations increased significantly with age when the four groups were pooled and in $\mathrm{AG}, \mathrm{RG}$ and $\mathrm{CON}$ when groups were individualized. A decrease in OPG with age was only observed in RG.

\section{Discussion}

This cross-sectional study demonstrates that during the growth period, only physical activities that generate high
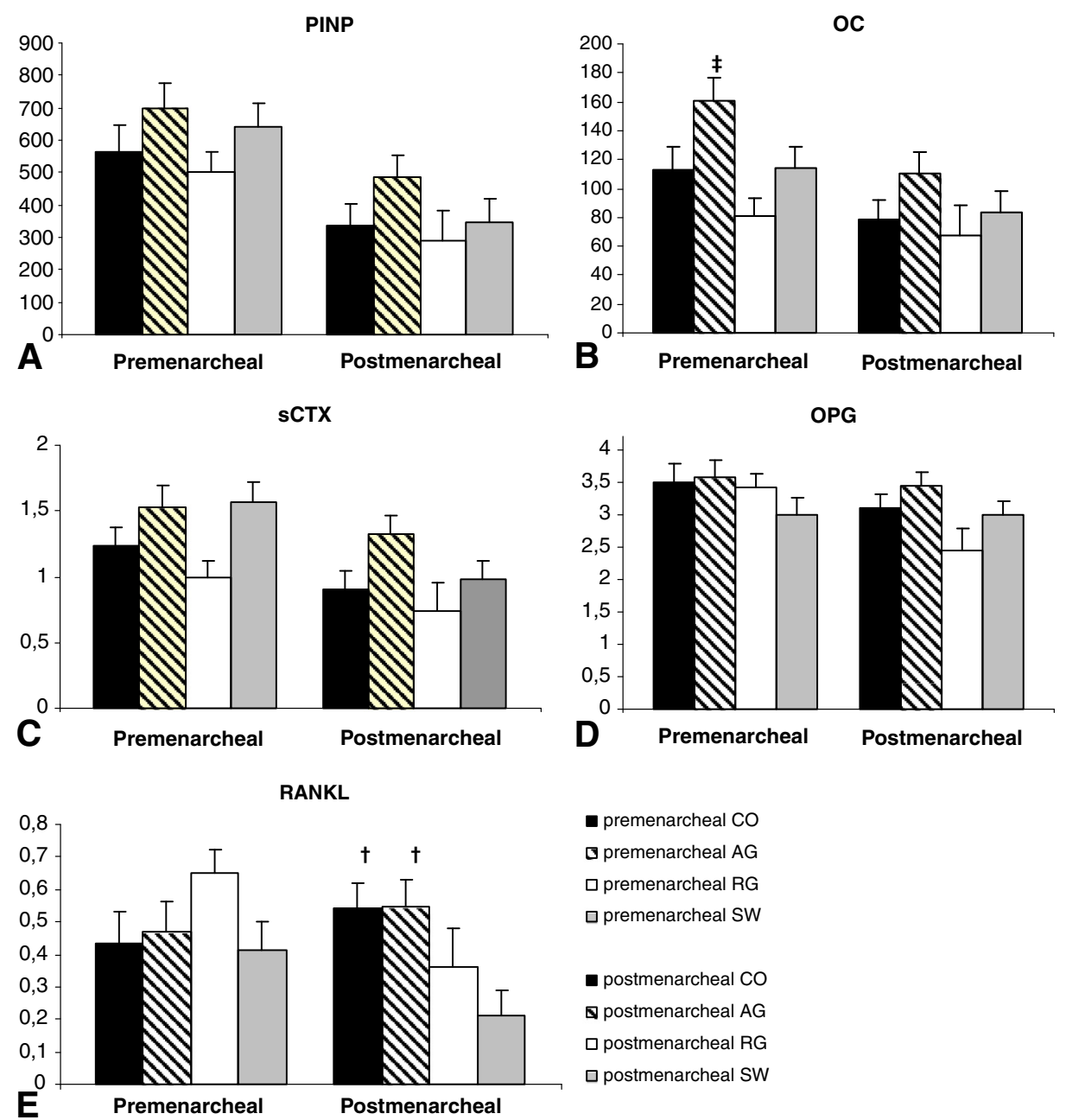

Fig. 2 - Adjusted bone biochemical markers and OPG/RANKL concentrations by age in athletes and controls according to menarcheal status. Values are presented as means \pm SEM. Procollagen type I N-terminal propeptide: PINP (A), osteocalcin: OC (B), type I-C telopeptide breakdown products: sCTX (C), osteoprotegerin: OPG (D), and rank-ligand: RANKL (E). †p < 0.05 denotes a significant difference with swimmers; $\neq p<0.05$ denotes a significant difference with rhythmic gymnasts. 
mechanical strain improve aBMD and bone geometry. The inclusion of subjects with a broad age range, from 10 to 18 years, in the same work showed that differences between sports with regard to bone status are present from an early age and up to the end of puberty. Nevertheless, these differences seem to be more marked after menarche.

This comparison of three sports generating different mechanical loads demonstrated the gradation and localization of the bone response according to the type of activity. Artistic gymnasts, who perform an intensive activity with high weight-bearing and impact (3.6 to 10.4 times body weight) [9] presented higher aBMD values than controls, as previously reported [8]. This improved aBMD was observed at each bone site in trabecular and cortical bone, as well as in upper and lower limbs. Our results confirm previous studies [22-24], but also provide additional information since puberty and the adolescent period were investigated for the first time. Moreover, the swimmers, who performed a no-impact activity, showed the same bone profile as CON and had lower values than AG. Our results thus strongly suggest that impact is essential to induce bone mass adaptation and that muscle contraction, which is the principal component of swimming, is not in itself sufficient $[3,12,25]$. The lack of a swimming effect on bone mass appeared to be independent of age or menarcheal status, as well as gender, as we demonstrated the lack of effect in adult male swimmers in an earlier study [26].

In our study, the difference in aBMD between groups was mainly observed in the postmenarcheal period. However, this key result was obtained in rather small subgroups, which is a limitation of this study, and thus interpretation requires great cautiousness. Nevertheless, many factors may explain this age-dependent bone adaptation. First, it is probable that a minimum number of years of training is necessary to induce additional bone mass gain in a context of already high bone mass acquisition, and the aBMD measured in the postmenarcheal period is in part the result of the cumulative effects of training throughout childhood. Second, as we have previously suggested [8], the peripubertal period is particularly favorable to additional bone acquisition because endocrine variations that may potentiate the action of mechanical strain on bone are observed [13,27]. In particular, the increase in IGF-1 and estradiol $\left(E_{2}\right)$ levels that occurs in this period has an essential concerted action on bone accretion during this period in untrained subjects [28], as well as in athletes $[29,30]$. In addition to menstrual subgroup analysis, it would also have been pertinent to determine the potential role of menstrual disorders on bone mass acquisition by comparing athletes with and without these disorders within the same group. Unfortunately, the very low number of subjects per group did not allow us to do so here.

AG and RG have already been compared in a few studies, but with a limited number of subjects within a narrow age class and limited training [23,32]. Nevertheless, a comparative approach is fruitful because, although these two sports are similar in terms of high-volume training at a very early age, dietary restriction, high prevalence of menstrual disorders, and bone maturation delay as observed here or in previous reports $[17,18]$, they differ in terms of mechanical loading and individual growth poten- tial $[17,18]$. Moreover, AG imposes a great mechanical load on the upper arms, principally due to asymmetric bar exercise, and the lower limbs and trunk [31], whereas RG requires less arm and body strength, loading mostly at the lower limbs [23,32]. This may be a causal factor of the local aBMD differences observed at the upper limbs and specifically at the radius. Interestingly, our results confirmed some previous data [23], but differed from other findings $[32,33]$. This slight discrepancy may be related to the age of the subjects and/or their menarcheal status, as demonstrated by our evaluation in subgroups according to this status. At the lower limbs and femoral region, the lack of an aBMD difference between AG and RG suggests that a threshold of solicitation is sufficient to induce maximal bone mass gain, beyond which no substantial gain is obtained. It is interesting to note that the osteogenic effect in $A G$ and $R G$ was observed even though both groups presented bone maturation retardation and delayed menarche, factors known to have negative effects on bone mass acquisition [34].

In parallel to the findings for bone density, the bone geometry evaluated by HSA at the proximal femur was also modified according to the type of physical activity. For the two groups of gymnasts and especially AG, an adaptation of bone geometry to high mechanical strain was demonstrated by higher values for CSA (cross-sectional area occupied by bone) and mean cortical thickness compared with SW and CO. Among the determinants of bone strength, cortical thickness plays a crucial role [35]. This was demonstrated in animal studies, where an increase in cortical thickness by inhibitors of bone resorption treatment contributed to the improvement in bone strength [36]. Moreover, these modifications were associated with a greater index of strength in binding $(Z)$ and a lower value for local cortical buckling under compressive loads (buckling ratio). We previously suggested that the higher values of CSA and mean cortical thickness observed in AG without modification in width are probably due to corticalization of the trabecular structure beneath the endocortical surface [8]. Corticalization has also been reported in young female athletes and postmenopausal women participating in a strength-training program $[37,38]$. The evaluation of the bone structure in RG tends to support this hypothesis because endocortical diameter was significantly decreased in these athletes, who also perform under high mechanical strain. The HSA technique was particularly pertinent in this situation because we were able to demonstrate that femoral geometry may differ between AG and RG even when aBMD in the proximal femoral region shows no difference. Endocortical diameter and width showed the biggest variation, with RG femur geometry appearing schematically "stockier" than in AG. This geometry may confer specific biomechanical properties, as section modulus increased only in AG, perhaps conferring an increase in bone strength in this group alone.

Few studies have reported using the HSA method in children or adolescent athletes. In premenarcheal gymnasts with a mean training volume of $15 \mathrm{~h} / \mathrm{wk}$, greater values for CSA and section modulus were reported [22], confirming that the bone geometry of the proximal femur adapted to the loading imposed by this sport. More recently, Ferry et al. [12] compared adolescent female swimmers and soccer players 
and reported that the girls playing the high-impact sport of soccer exhibited greater cortical thickness than swimmers due to increased bone section (width). The authors suggested that the "unloading" environment of the swimming pool may have a deleterious effect on bone geometry. We observed no alteration in the bone geometry of swimmers as compared with age-paired controls. The discrepancy between our data and Ferry's may be related to the training volume (mean $14 \mathrm{~h} /$ wk vs. $10 \mathrm{~h} / \mathrm{wk}$ ), the type of training (associated with or without resistance training), the age of the participants, and the number of years of practice. It is possible that the normal/ low aBMD associated with normal bone geometry conferred an advantage in terms of buoyancy for these athletes $[39,40]$. The HSA results seem particularly pertinent in sport because they reinforce those obtained by direct evaluation of bone geometry such as PQCT at the tibial cortical site in premenarcheal rhythmic gymnasts [18]. Independently, the modification in bone geometry and strength as evaluated by pQCT was also observed in artistic gymnasts at the forearm [41].

We found a significant decrease in bone remodeling markers (OC, PINP, CTX) with age, as generally observed in inactive children $[42,43]$. Consequently, the concentrations were higher in the premenarcheal than postmenarcheal period, but the variation between the two periods followed the same profile in the four groups. Only RG presented low bone remodeling (OC and CTX) compared with AG when groups were subdivided according to menarcheal status, although the groups did not differ in terms of bone density. The long-term effects of physical activity on bone remodeling in children are very difficult to identify $[18,44]$. Although we followed the recommendations for using bone markers [14], the cross-sectional evaluation may have been a limitation because many parameters interfere with bone remodeling in sports (period in the training season, duration of recovery before sampling, etc.). Nevertheless, a longitudinal study also reported no specific difference between gymnasts and controls before and after 6 months of training [44].

The effect of physical activity on the OPG/RANKL system has received little attention $[15,16,45]$, particularly during the peripubertal period [8]. RANKL concentrations were low in SW compared with the other groups, which showed values increasing with age. Our data confirmed previous results in controls [46], suggesting that several years of athletic training do not modify the physiological variation. Conversely, it was surprising to find no modification in OPG levels whatever the menarcheal status or type of sport, since in vitro studies have demonstrated that mechanical loading increases OPG $[47,48]$. However, the effects of physical activity in humans are less straightforward. This was illustrated by the divergent variations in OPG levels in two studies of postmenopausal women, after 8- to 12-month training programs [49,50]. Comparative studies of runners and sedentary subjects also showed either similar or different OPG/RANKL profiles $[15,16]$. In our study, the finding that the type of sport or the aBMD did not help to draw specific OPG/RANKL profiles suggests that this system is not the main factor of bone adaptation. It is also likely that after many years of training, a new equilibrium is achieved.

The main strength of the present study is that we were able to investigate a broad spectrum of physical activities gener- ating different mechanical loadings on bone. Moreover, the wide age range covering pre- to post-menarcheal periods suggested that bone adaptation may be dependent on the degree of sexual maturity. The use of several physical and biological techniques of investigation further ensured a thorough characterization of the variations in bone tissue in terms of bone mass, bone geometry and bone remodeling. However, some of the limitations of this cross-sectional study should be noted, as well. First, the participants were not investigated longitudinally, which may have resulted in interindividual variations that limit our conclusions. Nevertheless, our population was carefully age-paired, and within each group all athletes had comparable training status. Second, the observation of the major difference between groups postmenarche should be interpreted with caution because the number of subjects in each subgroup was low.

In conclusion, our results suggest that during the growth period, only high-impact activities enhance peak bone mass acquisition and bone geometry in athletes. The benefit to bone health seems to be dependent on age and menarcheal status and independent of the OPG/RANKL system. Moreover, the association of the DXA and HSA methods demonstrates that bone geometry may differ for the same aBMD, conferring specific bone strength. The results observed in gymnasts may not be found systematically in other groups of athletes, especially in elite gymnasts whose strict dietary restrictions and the frequent negative energy balance may have a detrimental effect on bone health.

\section{Author contributions}

Laurent Maïmoun (LM); Olivier Coste (OC), Pascal Philibert (PP); Karine Briot (KB); Thibault Mura (TB); Florence Galtier (FG); Denis Mariano-Goulart (DMG), Françoise Paris (FP); Charles Sultan (CS).

Study design: LM, OC, FP and CS. Study conduct: LM, OC, PP, FG, KB, DMG and FP. Data collection: LM and OC. Data analysis: LM and TM. Data interpretation: LM, OC, PP, FP and CT. Manuscript preparation: LM, OC, PP, TM, FG, KB, DMG, FP and CS. Revision of manuscript content: LM, OC, PP, TM, FG, KB, DMG, FP and CS. Final approval of the manuscript: LM, OC, PP, TM, BC, FG, KB, DMG, FP and CS. LM and TM take responsibility for the integrity of the data analysis.

\section{Acknowledgments}

The authors would like to express their thanks to the athletes and their parents for their participation. We thank also MarieAgnès Martin for her excellent technical assistance, and Roche Diagnostics and Immunodiagnostic Systems for providing the biochemical kits.

\section{Conflict of interest}

The authors declare no conflict of interest. 
[1] Frost HM, Schonau E. The "muscle-bone unit" in children and adolescents: a 2000 overview. J Pediatr Endocrinol Metab 2000;13:571-90.

[2] Grimston SK, Willows ND, Hanley DA. Mechanical loading regime and its relationship to bone mineral density in children. Med Sci Sports Exerc 1993;25:1203-10.

[3] Courteix D, Lespessailles E, Peres SL, et al. Effect of physical training on bone mineral density in prepubertal girls: a comparative study between impact-loading and non-impactloading sports. Osteoporos Int 1998;8:152-8.

[4] McCreadie BR, Goldstein SA. Biomechanics of fracture: is bone mineral density sufficient to assess risk? J Bone Miner Res 2000;15:2305-8.

[5] van der Meulen MC, Jepsen KJ, Mikic B. Understanding bone strength: size isn't everything. Bone 2001;29:101-4.

[6] Sayers A, Mattocks C, Deere K, et al. Habitual levels of vigorous, but not moderate or light, physical activity is positively related to cortical bone mass in adolescents. J Clin Endocrinol Metab 2011;96:793-802.

[7] Beck TJ, Ruff CB, Warden KE, et al. Predicting femoral neck strength from bone mineral data. A structural approach. Invest Radiol 1990;25:6-18.

[8] Maimoun L, Coste O, Mariano-Goulart D, et al. In peripubertal girls, artistic gymnastics improves areal bone mineral density and femoral bone geometry without affecting serum OPG/RANKL levels. Osteoporos Int 2011;22:3055-66.

[9] Daly RM, Rich PA, Klein R, Bass S. Effects of high-impact exercise on ultrasonic and biochemical indices of skeletal status: a prospective study in young male gymnasts. J Bone Miner Res 1999;14:1222-30.

[10] Maimoun L, Mariano-Goulart D, Couret I, et al. Effects of physical activities that induce moderate external loading on bone metabolism in male athletes. J Sports Sci 2004;22: 875-83.

[11] Morel J, Combe B, Francisco J, Bernard J. Bone mineral density of 704 amateur sportsmen involved in different physical activities. Osteoporos Int 2001;12:152-7.

[12] Ferry B, Duclos M, Burt L, et al. Bone geometry and strength adaptations to physical constraints inherent in different sports: comparison between elite female soccer players and swimmers. J Bone Miner Metab 2011;29:342-51.

[13] Hind K, Burrows M. Weight-bearing exercise and bone mineral accrual in children and adolescents: a review of controlled trials. Bone 2007;40:14-27.

[14] Maimoun L, Sultan C. Effects of physical activity on bone remodeling. Metabolism 2011;60:1655-63.

[15] Herrmann M, Herrmann W. The assessment of bone metabolism in female elite endurance athletes by biochemical bone markers. Clin Chem Lab Med 2004;42:1384-9.

[16] Ziegler S, Niessner A, Richter B, et al. Endurance running acutely raises plasma osteoprotegerin and lowers plasma receptor activator of nuclear factor kappa B ligand. Metabolism 2005;54:935-8.

[17] Georgopoulos NA, Markou KB, Theodoropoulou A, et al. Growth retardation in artistic compared with rhythmic elite female gymnasts. J Clin Endocrinol Metab 2002;87: 3169-73.

[18] Tournis S, Michopoulou E, Fatouros IG, et al. Effect of rhythmic gymnastics on volumetric bone mineral density and bone geometry in premenarcheal female athletes and controls. J Clin Endocrinol Metab 2011;95: 2755-62.

[19] Maimoun L, Coste O, Galtier F, et al. Bone mineral density acquisition in peripubertal female rhythmic gymnasts is directly associated with plasma IGF1/IGF-binding protein 3 ratio. Eur J Endocrinol 2010;163:157-64.
[20] Maimoun L, Coste O, Jaussent A, et al. Bone mass acquisition in female rhythmic gymnasts during puberty: no direct role for leptin. Clin Endocrinol (Oxf) 2010;72:604-11.

[21] Tanner J. Growth at adolescence. Oxford: Blackwell Scientific Publications; 1962.

[22] Greulich WW, Pyle SI. Radiographic atlas of skeletal development of hand and wrist. . 2nd Ed.Stanford, CA: Stanford University Press; 1959.

[23] Khoo BC, Beck TJ, Qiao QH, et al. In vivo short-term precision of hip structure analysis variables in comparison with bone mineral density using paired dual-energy x-ray absorptiometry scans from multi-center clinical trials. Bone 2005;37: 112-21.

[24] Faulkner RA, Forwood MR, Beck TJ, et al. Strength indices of the proximal femur and shaft in prepubertal female gymnasts. Med Sci Sports Exerc 2003;35:513-8.

[25] Helge EW, Kanstrup IL. Bone density in female elite gymnasts: impact of muscle strength and sex hormones. Med Sci Sports Exerc 2002;34:174-80.

[26] Taaffe DR, Robinson TL, Snow CM, Marcus R. High-impact exercise promotes bone gain in well-trained female athletes. J Bone Miner Res 1997;12:255-60.

[27] Nikander R, Sievanen H, Heinonen A, Kannus P. Femoral neck structure in adult female athletes subjected to different loading modalities. J Bone Miner Res 2005;20:520-8.

[28] Maimoun L, Lumbroso S, Manetta J, et al. Testosterone is significantly reduced in endurance athletes without impact on bone mineral density. Horm Res 2003;59:285-92.

[29] Frost HM. The role of changes in mechanical usage set points in the pathogenesis of osteoporosis. J Bone Miner Res 1992;7: 253-61.

[30] Xu L, Wang Q Lyytikainen A et al. Concerted actions of insulin-like growth factor 1, testosterone, and estradiol on peripubertal bone growth: a 7-year longitudinal study. J Bone Miner Res 26: 2204-11.

[31] Markou KB, Mylonas P, Theodoropoulou A, et al. The influence of intensive physical exercise on bone acquisition in adolescent elite female and male artistic gymnasts. J Clin Endocrinol Metab 2004;89:4383-7.

[32] Vicente-Rodriguez G, Dorado C, Ara I, et al. Artistic versus rhythmic gymnastics: effects on bone and muscle mass in young girls. Int J Sports Med 2007;28:386-93.

[33] Robinson TL, Snow-Harter C, Taaffe DR, et al. Gymnasts exhibit higher bone mass than runners despite similar prevalence of amenorrhea and oligomenorrhea. J Bone Miner Res 1995;10:26-35.

[34] Chevalley T, Bonjour JP, Ferrari S, Rizzoli R. Deleterious effect of late menarche on distal tibia microstructure in healthy 20-year-old and premenopausal middle-aged women. J Bone Miner Res 2009;24:144-52.

[35] Turner CH. Biomechanics of bone: determinants of skeletal fragility and bone quality. Osteoporos Int 2002;13: 97-104.

[36] Ammann P, Rizzoli R, Meyer JM, Bonjour JP. Bone density and shape as determinants of bone strength in IGF-I and/or pamidronate-treated ovariectomized rats. Osteoporos Int 1996;6:219-27.

[37] Adami S, Gatti D, Braga V, et al. Site-specific effects of strength training on bone structure and geometry of ultradistal radius in postmenopausal women. J Bone Miner Res 1999;14:120-4.

[38] Nikander R, Sievanen H, Uusi-Rasi K, et al. Loading modalities and bone structures at nonweight-bearing upper extremity and weight-bearing lower extremity: a pQCT study of adult female athletes. Bone 2006;39:886-94.

[39] Taaffe DR, Snow-Harter C, Connolly DA, et al. Differential effects of swimming versus weight-bearing activity on bone mineral status of eumenorrheic athletes. J Bone Miner Res 1995;10:586-93. 
[40] Cassell C, Benedict M, Specker B. Bone mineral density in elite 7- to 9-yr-old female gymnasts and swimmers. Med Sci Sports Exerc 1996;28:1243-6.

[41] Burt LA, Naughton GA, Greene DA, Ducher G. Skeletal differences at the ulna and radius between pre-pubertal nonelite female gymnasts and non-gymnasts. J Musculoskelet Neuronal Interact 2011;11:227-33.

[42] Cadogan J, Blumsohn A, Barker ME, Eastell R. A longitudinal study of bone gain in pubertal girls: anthropometric and biochemical correlates. J Bone Miner Res 1998;13: 1602-12.

[43] Mora S, Pitukcheewanont P, Kaufman FR, et al. Biochemical markers of bone turnover and the volume and the density of bone in children at different stages of sexual development. J Bone Miner Res 1999;14:1664-71.

[44] Nickols-Richardson SM, O'Connor PJ, Shapses SA, Lewis RD. Longitudinal bone mineral density changes in female child artistic gymnasts. J Bone Miner Res 1999;14:994-1002.

[45] Kerschan-Schindl K, Thalmann M, Sodeck GH, Skenderi K, et al. A $246-\mathrm{km}$ continuous running race causes significant changes in bone metabolism. Bone 2009;45: 1079-83.

[46] Wasilewska A, Rybi-Szuminska AA, Zoch-Zwierz W. Serum osteoprotegrin (OPG) and receptor activator of nuclear factor kappaB (RANKL) in healthy children and adolescents. J Pediatr Endocrinol Metab 2009;22:1099-104.

[47] Kobayashi Y, Hashimoto F, Miyamoto H, et al. Force-induced osteoclast apoptosis in vivo is accompanied by elevation in transforming growth factor beta and osteoprotegerin expression. J Bone Miner Res 2000;15:1924-34.

[48] Kim CH, You L, Yellowley CE, Jacobs CR. Oscillatory fluid flowinduced shear stress decreases osteoclastogenesis through RANKL and OPG signaling. Bone 2006;39:1043-7.

[49] Marques EA, Wanderley F, Machado L, et al. Effects of resistance and aerobic exercise on physical function, bone mineral density, OPG and RANKL in older women. Exp Gerontol 2011;46:524-32.

[50] Bergstrom I, Parini P, Gustafsson SA, et al. Physical training increases osteoprotegerin in postmenopausal women. J Bone Miner Metab 2011;30:202-7. 